\title{
A Common Optical Approach to Thickness Optimization in Polymer and Perovskite Solar Cells
}

\section{Olga lakobson}

Frumkin Institute of Physical Chemistry and Electrochemistry

Oxana Gribkova

Frumkin Institute of Physical Chemistry and Electrochemistry

\section{Alexey Tameev}

National Research University Higher School of Economics

Jean-Michel Nunzi ( $\nabla$ nunzijm@queensu.ca )

Department of Chemistry, and Department of Physics, Engineering Physics and Astronomy, Queen's University, Kingston ON K7L-3N6, Canada

\section{Research Article}

Keywords: organic bulk heterojunction, light absorbance and exciton generation rate, photoactive materials

Posted Date: December 7th, 2020

DOI: https://doi.org/10.21203/rs.3.rs-115441/v1

License: (c) (i) This work is licensed under a Creative Commons Attribution 4.0 International License. Read Full License

Version of Record: A version of this preprint was published at Scientific Reports on March 2nd, 2021. See the published version at https:// doi.org/10.1038/s41598-021-84452-x. 


\title{
A common optical approach to thickness optimization in polymer and perovskite solar cells
}

\author{
Olga D. Iakobson ${ }^{1}$, Oxana L. Gribkova ${ }^{1}$, Alexey R. Tameev ${ }^{1,2, \dagger}$, Jean-Michel Nunzi ${ }^{3, \dagger}$ \\ ${ }^{1}$ Frumkin Institute of Physical Chemistry and Electrochemistry of the Russian Academy \\ of Sciences, 31 bld.4 Leninsky Prosp., Moscow 119071, Russian Federation \\ ${ }^{2}$ National Research University Higher School of Economics, 20 Myasnitskaya Str., \\ Moscow 101000, Russian Federation \\ ${ }^{3}$ Department of Chemistry, and Department of Physics, Engineering Physics and \\ Astronomy, Queen's University, Kingston ON K7L-3N6, Canada \\ † tameev@elchem.ac.ru; a.tameev@gmail.com
}

\$nunzijm@queensu.ca

\begin{abstract}
The structure of experimentally designed solar cells was optimized in terms of the photoactive layer thickness for both organic bulk heterojunction and hybrid perovskite solar cells. The photoactive layer thickness had a totally different behavior on the performance of the organic and hybrid solar cells. Analysis of the optical parameters using transfer matrix modeling within the Maxwell-Garnett effective refractive index model shows that light absorbance and exciton generation rate in the photoactive layer can be used to optimize the thickness range of the photoactive layer. Complete agreement between experimental and simulated data for solar cells with photoactive materials that have very different natures proves the validity of the proposed modeling method.
\end{abstract}

\section{Introduction}

Solar cells (SCs) attract researchers attention as promising renewable energy sources. Two perspective types of SC are currently under intensive investigation. First one is based on organic photoactive materials, which advantages include mechanical flexibility, light weight, and low fabrication cost into large-area devices [1-3]. The largest reported power conversion efficiency of organic solar cells is based on the bulk-heterojunction (BHJ) concept [1]. The bulk heterojunction provides not only high surface contacts for charge separation, but also an interpenetrating network for efficient charge transport [1]. Second type of SCs that recently succesfully entered into the field of photovoltaics is based on metal-organic compounds with the perovskite structure. Perovskite materials are characterized by large absorption coefficients, relatively high carrier mobility, long carrier lifetime, as well as simple fabrication process, which result in the development of highly efficient perovskite solar cells [4]. 
One challenge in modern solar energy is the development of cost-effective devices with facile device structure optimization. Optimization requires proper understanding of the physical procesess that underlie device performance [5].

There have been several efforts to understand the device operation mechanism of organic SCs. The effects of variables such as morphology, temperature, light intensity, and optical absorption on the device performance, and primarily on the short circuit current characteristics, has been studied extensively $[1,6]$.

In addition to experimental approaches in which optimization sometimes proceeds through trial and fail, attempts to analize device performances through simulation models has shown some success $[1-3,7]$. There are two general approaches in SC simulation that take into account optical (incoupling of the light to the multilayer stack) [1-3,7] or electrical (extraction of charges) [2,3] parameters of the device materials, and sometimes combinations of both [6,8]. However, electrical models can be quite combersome computationally [2].

Influence of the optical effects on the performance of SCs is usually derived from the device design features since the thickness of thin films in SCs is generally smaller than the wavelength of the incident light [6]. The objective of optical modeling and simulation is to calculate the light absorption and exciton creation based on the materials properties and structure of the solar cells [6]. Several model were implemented to analyze light absorbing. In [9] Peterson et al. used the transfer matrix formalism to calculate the absorbed optical energy in a multilayer structure, which turned out to agree with their experimental data. The model considers isotropic layers [6], which may fail in predicating the performances of hybrid perovskite solar cells owing to their microcrystalline structure. Optical modeling was also recently applied to the optimization of the photoactive layer thickness in hybrid perovskite SC [4].

In the present work, we successfully applied known optical simulation methods to analyze the performance of solar cells built from completely different materials. A newly engineered hole transport material superior than commonly used PEDOT-PSS: a polyaniline complexed with a polyacid, was employed in this study. As a limited number of works was devoted to a direct comparison of experimental and modeling results, the question whether it is possible to extend a given model to other materials is still open. Full aggrement between simulated and experimental results allows us to conclude that the model can be used as a general predictive tool to optimize devices constructed from different materials. This simple approach offers the opportunity to rapidly test and validate novel device concepts. It avoids the lengthy fabrication of large sets of prototypes which are usually required for an experimental optimization [3].

\section{Experimental part}


Solar cells (SC) designed here and based on an organic or hybrid photoactive layer (PAL) with the structure: ITO (indium tin oxide) on glass (anode)/ hole transporting layer (HTL)/ photoactive layer (PAL)/ electron transporting layer (ETL) / Al (cathode).

ITO $(100 \mathrm{~nm})$ covered glass with $15 \mathrm{Ohm} / \mathrm{square}$ sheet resistance (Kintec) served as anode. A drop-casted film of polyaniline complex with poly(2-acrylamido-2-methyl-1-propanesulfonic acid) (PANI-PAMPSA) obtained as described earlier $[10,11]$ was used as HTL.

For the organic PAL, a chlorobenzene mixture of regioregular P3HT polymer (4002-EE; Rieke Metals) and fullerene derivative $\mathrm{PC}_{71} \mathrm{BM}$ electron-acceptor (SES Research) (1:0.8 wt.) was used. Details of the PAL preparation can be found in [12]. The organic PAL thickness was varied by tuning the speed and time of centrifugation ( 1500 vs $900 \& 2000 \mathrm{rpm}$ and 60 vs $60 \& 45$ s). The $\mathrm{LiF}$ electron transporting layer $(0.9 \mathrm{~nm})$ and $\mathrm{Al}$ cathode $(80 \mathrm{~nm})$ were deposited on the PAL by thermal evaporation under $\sim 10^{-6}$ mbar vacuum in an Auto500 Edwards Evaporator.

The hybrid perovskite $\mathrm{SC}$ was based on a methylammonium lead iodide $\left(\mathrm{MAPbI}_{3}\right) \mathrm{PAL}$. A N, N-dimethylformamide mixture of methylammonium iodide (Dyesol) and lead iodide (AlfaAesar) at different concentrations was deposited on the HTL by centrifugation ( $5000 \mathrm{rpm}, 30$ s). The $\mathrm{MAPbI}_{3}$ perovskite was formed by the method described earlier [13]. A Fullerene $\mathrm{C}_{60}$ electron transporting layer (40 nm), a 2,9-dimethyl-4,7-diphenyl-1,10-phenanthroline hole blocking layer $(7 \mathrm{~nm})$ and $\mathrm{Al}$ cathode $(80 \mathrm{~nm})$ were deposited on the PAL by thermal evaporation under $\sim 10^{-6}$ mbar vacuum in an Auto500 Edwards Evaporator.

Thickness of all layers was determined using a KLA-Tencor D-100 Profiler.

Photovoltaic performances were studied inside a glovebox using a Keithley 2400 SMU under the AM1.5 illumination of an Oriel solar simulator (Xe lamp $150 \mathrm{~W}$, Newport Corp.).

Optical parameters of the organic and hybrid SCs were studied by modeling the photocurrent action spectra using the $2 \times 2$ optical transfer matrix method [9]. The method consists in representing the SC as a set of flat layers. Each one is characterized by its thickness and complex refractive index $\tilde{n}=\eta+i \kappa$, within the Maxwell-Garnett model [1].

The following parameters were assessed from modeling: light absorbance in the PAL, the Shockley-Queisser limit at the real SC efficiency and the exciton generation rate in the PAL were assessed from modeling. Light absorption in the PAL was determined by:

$$
\int_{\lambda_{\text {down }}}^{\lambda_{\text {do }}} A(\lambda) \cdot S_{\lambda} \cdot d \lambda
$$

where $\lambda$ is the wavelength, $A(\lambda)$ is absorption coefficient, $S(\lambda)$ is radiation power density normalized under AM1.5G condition with $S(\lambda) \mathrm{d} \lambda=1$. Shockley-Queisser limit at the real SC efficiency was determined as: 


$$
10^{-9} \cdot \int_{\lambda_{\text {down }}}^{\lambda_{u p}} \frac{A(\lambda)}{h \cdot c} \cdot \lambda \cdot E_{g} \cdot S_{\lambda} \cdot d \lambda
$$

where $E_{g}$ is the band gap, $h$ the Planck's constant, $c$ the speed of light in vacuum. Exciton generation rate in the PAL was determined as [14]:

$$
G=\int_{0}^{d} G(z) d z=\int_{0}^{d}\left(\int_{0}^{\infty} S(\lambda) \gamma(\lambda) \frac{\lambda}{h c} Q_{\lambda}(z) d \lambda\right) d z
$$

where $d$ is the PAL thickness, $G(z)$ the exciton rate at normal incidence at coordinate $\mathrm{z}, \gamma(\lambda)$ is photon to exciton conversion efficiency and $Q_{\lambda}(z)$ is the average energy flow dissipation.

Complex refractive indices were determined by analysis of the spectroscopic ellipsometry data obtained either experimentally using a UVISEL2 elipsometer for ITO glass, PANI-PAMPSA and $\mathrm{MAPbI}_{3}$ or from the literature [15] for the BHJ PAL, [16] C60, [17] and Al. Measured optical parameters provide a more accurate optical sumulation [1]. Simulation was performed in the 360 $\mathrm{nm}$ to $800 \mathrm{~nm}$ spectral range.

\section{Results and discussion}

Light harvesting as well as density of generated excitons in the PAL vary along the layer thickness, which affects the short-circuit current $\left(J_{s c}\right)$ and in consequence the power conversion efficiency (PCE). Optimization of the functional layer thickness is therefore one of the routes to increase the PCE $[3,4,7,18]$. However, values of the optimal thickness will be specific of each particular system within a selected SC structure and of the materials used for the PAL. However, provided that the processes undelying device physics are the same for different PALs, it is expected to find similar explanations to the observed dependences. Among the abundance of scattered exprimental data, no general model taking into account the physical parameters of the layers forming the SCs was proposed. Such model would make it possible to predict the dependence of the device efficiency on the layer thickness and thus to establish the optimal thickness range. We have studied the influence of PAL thickness on the power conversion efficiency (PCE) with two entitely different photoactive materials. One of them is the widely studied $\mathrm{P} 3 \mathrm{HT}-\mathrm{PC}_{71} \mathrm{BM}$ polymer composition which forms a bulk heterojunction (BHJ) in the PAL. The other one is the intensively studied methylammonium lead iodide $\left(\mathrm{MAPbI}_{3}\right)$ perovskite which is an organic-inorganic (hybrid) microcrystalline material.

Influence of the P3HT-PC ${ }_{71} \mathrm{BM}$ and of other BHJ PAL thickness on the PCE of devices with a conventional PEDOT-PSS HTL was experimentally studied elsewhere [1,2,19-25]. The 
polyaniline complex with a polyacid (PANI-PAMPSA) used here as an HTL already demonstrated some advantages over PEDOT-PSS, like the absence of an heterophase formation in dispersion during storage and the hygroscopicity of the layer, a resonable electrical conductivity [26,27], and the stability of electrical and optical properties for at least 2 years [28]. Moreover, organic SCs with the same structure bearing an HTL made of either PANI-PAMPSA or PEDOT-PSS showed similar performances [29].

Table 1. Dependence of the short-circuit current density $(J S c)$ and power conversion efficiency $(P C E)$ on the $\mathrm{P} 3 \mathrm{HT}-\mathrm{PC}_{71} \mathrm{BM}$ thickness.

\begin{tabular}{|c|c|c|}
\hline $\begin{array}{c}\text { P3HT-PC } \\
\text { thickness, } \mathrm{Bm}\end{array}$ & $J_{s c}, \mathrm{~mA} / \mathrm{cm}^{2}$ & PCE, \% \\
\hline 35 & 4.07 & 1.3 \\
\hline 55 & 5.85 & 1.9 \\
\hline 95 & 8.82 & 3.0 \\
\hline
\end{tabular}

Table 1 shows the dependence of the device parameters, JSc and PCE, on the P3HT$\mathrm{PC}_{71} \mathrm{BM}$ thickness for SC with a PANI-PAMPSA HTL. The thinnest P3HT-PC ${ }_{71} \mathrm{BM}$ layer is linited to $35 \mathrm{~nm}$ owing to the necessity of obtaining a continuous uniform thin film. The thicker the PAL, the larger $J S C$ and PCE. PCE increases more than twice within the studied range (Table 1). Further increase of the PAL thickness leads to a dramatic increase of the layer roughness. Moreover, while increasing the layer thickness would result in enhanced light harvesting, it also restricts charge extraction to the electrodes owing to the limited diffusion length of charge carriers which induces recombination losses [2,21,22,30]. Therefore, with PAL thicknesses larger than 100 $\mathrm{nm}$, most generated charge carriers recombine before reaching the electrode [2].

Thickness of the perovskite absorber is also an important parameter contributing to optimize solar cell performances [4]. A completely different picture was found for $\mathrm{MAPbI}_{3}$ based SCs (Table 2) with the PANI-PAMPSA complex also used as an HTL. There is not a clear dependence of the average performance parameters on the PAL thickness for the $\mathrm{MAPbI}_{3}$ devices (Table 2). The PCE ranges between $7 \%$ and $9 \%$ with about $8 \%$ variation. Variation of the perovskite layer thickness in the studied 350-500 nm range does not significantly affect the PCE. It is worth noting that the PCE in the studied devices corresponds to the reported analogues in the literature $[31,32]$. 
Table 2. Dependence of the short-circuit current density $(J S c)$ and power conversion efficiency $(P C E)$ on the $\mathrm{MAPbI}_{3}$ thickness.

\begin{tabular}{|c|c|c|}
\hline $\begin{array}{c}\mathrm{MAPbI}_{3} \text { thickness, } \\
\mathrm{nm}\end{array}$ & $J_{S c}, \mathrm{~mA} / \mathrm{cm}^{2}$ & PCE, $\%$ \\
\hline 350 & 15.00 & 7.6 \\
\hline 370 & 17.08 & 8.2 \\
\hline 390 & 14.62 & 8.0 \\
\hline 420 & 15.30 & 9.0 \\
\hline 450 & 14.04 & 7.1 \\
\hline 470 & 15.96 & 8.4 \\
\hline 490 & 17.62 & 8.3 \\
\hline
\end{tabular}

The transfer matrix method was applied to identify the physical origin of the observed dependences $[2,7,21,23,24,33]$, based on the optical energy absorbed in the multilayer structures [9]. Figure 1 shows the calculated optical parameters as a function of the PAL thickness for P3HT$\mathrm{PC}_{71} \mathrm{BM}$ (a) and $\mathrm{MAPbI}_{3}(\mathrm{~b})$.

As seen in Figure 1, the optical parameters demonstrate similar dependences within one type of PAL used. For the P3HT-PC ${ }_{71} \mathrm{BM}$ based PAL, the sharp increase of the calculated optical parameters with the increase of the PAL thickness till $\sim 80 \mathrm{~nm}$ is followed by their slight decrease to plateau level. So, the maximum PCE of the experimentally designed SC is likely to be explained by the combination of the highest rate of exciton generation and the maximum light absorption in the PAL at 90-100 nm thickness.

(a)

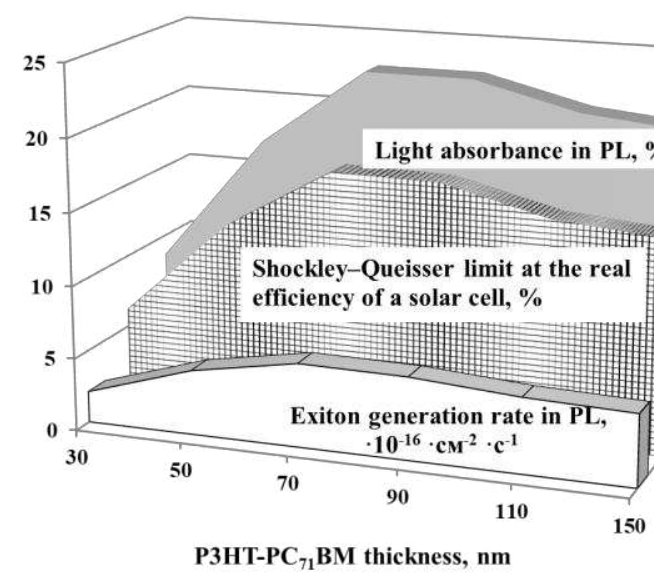

(b)

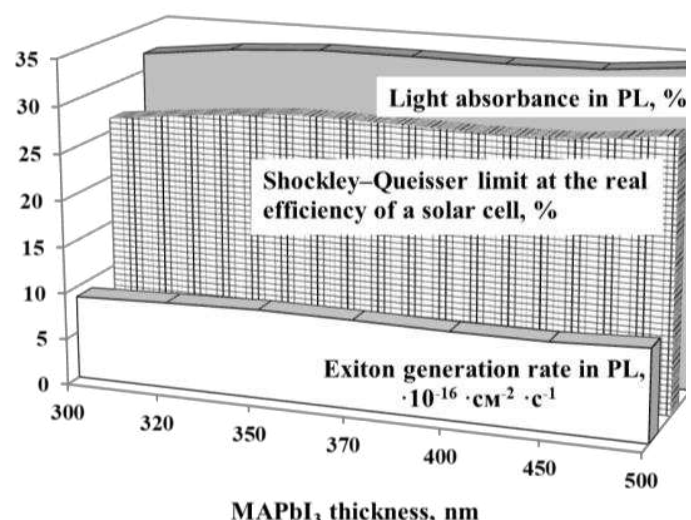

Figure 1. Calculated light absorbance in PAL, Shockley-Queisser limit at the real SC efficiency and exciton generation rate in the PAL for varied $\mathrm{P} 3 \mathrm{HT}-\mathrm{PC}_{71} \mathrm{BM}$ (a) and $\mathrm{MAPbI}_{3}$ (b) thickness. 
Oppositely, changes in the light absorbance, Shockley-Queisser limit at the real SC efficiency and exciton generation rate in the $\mathrm{MAPbI}_{3}$ based PAL with the increase of the PAL thickness are negligible. Therefore, the experimentally observed weak dependence of the PCE on the thickness of the perovskite PAL in the 350 to $500 \mathrm{~nm}$ range is explained by minor changes in both the light absorbance and the exciton generation rate.

The obtained results through modeling of the SC optical parameters agree with the experimental data obtained for the both types of PAL. Therefore, we could demonstrate that the same model concepts are applicable to assess the range of optimal parameters of the functional layers of SCs with different types of PAL. Obviously, the range of accessible layer thicknesses is governed by the thin film formation method. However, within the accessed experimental range, modeling with account of the optical parameters of the functional layers proves to be very efficient for specifying the range of thicknesses that ensure the maximum efficiency from the devices.

\section{Conclusion}

The matrix transfer method was used to explain theoretically the experimental effect of the thickness on the optical parameters of the layers and on the performance of SCs. We showed that the approach can be applied equally to the analysis of amorphous donor-acceptor systems and of microcrystalline structures. All of these were built with a water-dispersible polyaniline-polyacid complex as the hole transporting layer.

Although there has not been any pevious comprehensive study on the nature of the optical effects that are general enough to model SCs with completely different photoactive materials, our results show that optical modeling based on the transfer matrix method is applicable to solve the case. It provides an efficient method to validate proof-of-concepts regardless of the nature of the photoactive layers.

\section{ACKNOWLEDGEMENTS}

The research was financially supported by the Russian Science Foundation (project no. 18-1300409). A.R.T. is indebted to the Basic Research Program of the National Research University Higher School of Economics for the support. J.-M.N. acknowledges support from the Natural Science Research Council of Canada Discovery Grants (RGPIN-2020-07016)

\section{AUTHOR CONTRIBUTIONS}

O.D.I., numerical calculations, analysis of the data, preparation of the figures, writing and editing; O.L.G., synthesis of co-polymers and preparation of the films, writing and editing; 
A.R.T., conception, design and performance of the experiments, interpretation of the data, writing and editing, project administration; J.-M.N., conception, interpretation of the data, writing and editing.

\section{COMPETING INTERESTS}

The authors declare no competing interests.

\section{References:}

[1] D.W. Sievers, V. Shrotriya, Y. Yang, Modeling optical effects and thickness dependent current in polymer bulk-heterojunction solar cells, J. Appl. Phys. 100 (2006) 114509. doi:10.1063/1.2388854.

[2] M. Farrokhifar, A. Rostami, N. Sadoogi, Opto-Electrical Simulation of Organic Solar Cells, in: 2014 Eur. Model. Symp., IEEE, 2014: pp. 507-512. doi:10.1109/EMS.2014.73.

[3] F. Brioua, M. Remram, R. Nechache, H. Bourouina, Electrical and optical modeling of poly(3-hexylthiophene):[6,6]-phenyl- $\mathrm{C}_{61}$ butyric acid methyl ester P3HT-PCBM bulk heterojunction solar cells, Appl. Phys. A Mater. Sci. Process. 123 (2017) 704. doi:10.1007/s00339-017-1288-4.

[4] A.A. Kanoun, M.B. Kanoun, A.E. Merad, S. Goumri-Said, Toward development of highperformance perovskite solar cells based on $\mathrm{CH}_{3} \mathrm{NH}_{3} \mathrm{GeI}_{3}$ using computational approach, Sol. Energy. 182 (2019) 237-244. doi:10.1016/j.solener.2019.02.041.

[5] A. Moliton, J.M. Nunzi, How to model the behaviour of organic photovoltaic cells, Polym. Int. 55 (2006) 583-600. doi: 10.1002/pi.2038.

[6] G. Li, L. Liu, F. Wei, S. Xia, X. Qian, Recent progress in modeling, simulation, and optimization of polymer solar cells, IEEE J. Photovoltaics. 2 (2012) 320-340. doi: 10.1109/JPHOTOV.2012.2193385.

[7] E. Gondek, Optical optimization of organic solar cell with bulk heterojunction, OptoElectronics Rev. 22 (2014) 77-85. doi: 10.2478/s11772-014-0180-4.

[8] P. Peumans, A. Yakimov, S. R. Forrest, Small molecular weight organic thin-film photodetectors and solar cells, J. Appl. Phys. 93 (2003) 3693. doi: 10.1063/1.1534621.

[9] L.A.A. Pettersson, L.S. Roman, O. Inganäs, Modeling photocurrent action spectra of photovoltaic devices based on organic thin films, J. Appl. Phys. 86 (1999) 487-496. doi:10.1063/1.370757.

[10] O.L. Gribkova, A.A. Nekrasov, M. Trchova, V.F. Ivanov, V.I. Sazikov, A.B. Razova, V.A. Tverskoy, A. V. Vannikov, Chemical synthesis of polyaniline in the presence of poly(amidosulfonic acids) with different rigidity of the polymer chain, Polymer (Guildf). 
52 (2011) 2474-2484. doi:10.1016/j.polymer.2011.04.003.

[11] O.D. Omelchenko, O.L. Gribkova, A.R. Tameev, S.V. Novikov, A.V. Vannikov, Thin nanocomposite layers based on a complex of polyaniline and graphene, Prot. Met. Phys. Chem. Surf. 50 (2014) 613-619. doi: 10.1134/S207020511405013X.

[12] O.D. Iakobson, O.L. Gribkova, A.R. Tameev, A.A. Nekrasov, D.S. Saranin, A. Di Carlo, Graphene nanosheet/polyaniline composite for transparent hole transporting layer, J. Ind. Eng. Chem. 65 (2018) 309-317. doi:10.1016/j.jiec.2018.04.042.

[13] D.S. Saranin, V.N. Mazov, L.O. Luchnikov, D.A. Lypenko, P.A. Gostishev, D.S. Muratov, D.A. Podgorny, D.M. Migunov, S.I. Didenko, M.N. Orlova, D. V. Kuznetsov, A.R. Tameev, A. Di Carlo, Tris(ethylene diamine) nickel acetate as a promising precursor for hole transport layer in planar structured perovskite solar cells, J. Mater. Chem. C. 6 (2018) 6179-6186. doi:10.1039/C8TC01169A.

[14] R. Sh. Ikhsanov, I. E. Protsenko, A. V. Uskov, Increasing the efficiency of organic solar cells using plasmonic nanoparticles, Tech. Phys. Lett. 39 (2013) 450-453, doi: 10.1134/S1063785013050209.

[15] C. Stelling, C.R. Singh, M. Karg, T.A.F. König, M. Thelakkat, M. Retsch, Plasmonic nanomeshes: their ambivalent role as transparent electrodes in organic solar cells, Sci. Rep. 7 (2017) 42530. doi:10.1038/srep42530.

[16] P. Milani, M. Manfredini, G. Guizzetti, F. Marabelli, M. Patrini, Ellipsometric investigation of $\mathrm{C}_{60}$ single crystal, Solid State Commun. 90 (1994) 639-642. doi:10.1016/0038-1098(94)90537-1.

[17] K.M. McPeak, S. V. Jayanti, S.J.P. Kress, S. Meyer, S. Iotti, A. Rossinelli, D.J. Norris, Plasmonic Films Can Easily Be Better: Rules and Recipes, ACS Photonics. 2 (2015) 326333. doi:10.1021/ph5004237.

[18] K.O. Sylvester-Hvid, T. Ziegler, M.K. Riede, N. Keegan, M. Niggemann, A. Gombert, Analyzing poly(3-hexyl-thiophene):1-(3-methoxy-carbonyl)propyl-1-phenyl- (6,6) C61 bulk-heterojunction solar cells by UV-visible spectroscopy and optical simulations, J. Appl. Phys. 102 (2007) 054502. doi:10.1063/1.2775219.

[19] T. Ameri, G. Dennler, C. Waldauf, P. Denk, K. Forberich, M.C. Scharber, C.J. Brabec, K. Hingerl, Realization, characterization, and optical modeling of inverted bulkheterojunction organic solar cells, J. Appl. Phys. 103 (2008) 084506. doi:10.1063/1.2902804

[20] D. Lee, J. Kim, S. Noh, C. Lee, The thickness of active layer dependence of polymer solar cells, in: 10th IEEE Int. Conf. Nanotechnol., IEEE, 2010: pp. 1175-1178. doi:10.1109/NANO.2010.5698038. 
[21] L. Liu, G. Li, Thickness optimization of organic solar cells by optical transfer matrix, in: 2011 11th IEEE Int. Conf. Nanotechnol., IEEE, 2011: pp. 332-336. doi:10.1109/NANO.2011.6144456.

[22] I. Malti, A. Chiali, N.C. Sari, Numerical study of electrical behavior of P3HT/PCBM bulk heterojunction solar cell, Appl. Sol. Energy. 52 (2016) 122-127. doi:10.3103/S0003701X16020195.

[23] A.J. Moulé, J.B. Bonekamp, K. Meerholz, The effect of active layer thickness and composition on the performance of bulk-heterojunction solar cells, J. Appl. Phys. 100 (2006) 094503. doi:10.1063/1.2360780.

[24] R. Rahmani, H. Karimi, L. Ranjbari, M. Emadi, M. Seyedmahmoudian, A. Shafiabady, R. Ismail, Structure and Thickness Optimization of Active Layer in Nanoscale Organic Solar Cells, Plasmonics. 10 (2015) 495-502. doi:10.1007/s11468-014-9833-X.

[25] H. Movla, A. Mohammadalizad Rafi, N. Mohammadalizad Rafi, A model for studying the performance of P3HT:PCBM organic bulk heterojunction solar cells, Optik (Stuttg). 126 (2015) 1429-1432. doi:10.1016/j.ijleo.2015.04.020.

[26] O.D. Omelchenko, O.L. Gribkova, A.R. Tameev, A.V. Vannikov, The effect of the degree of graphene oxidation on the electric conductivity of nanocomposites based on a polyaniline complex, Tech. Phys. Lett. 40 (2014) 807-809. doi: 10.1134/S1063785014090260.

[27] O.D. Iakobson, O.L. Gribkova, A.R. Tameev, V. V. Kravchenko, A. V. Egorov, A. V. Vannikov, Conductive composites of polyaniline-polyacid complex and graphene nanostacks, Synth. Met. 211 (2016) 89-98. doi:10.1016/j.synthmet.2015.11.018.

[28] O.D. Iakobson, O.L. Gribkova, A.A. Nekrasov, V.A. Tverskoi, V.F. Ivanov, P.V. Mel'nikov, E.A. Polenov, A.V. Vannikov, Prot. Met. Phys. Chem. Surf. 52 (2016) 1005, doi: 10.1134/S207020511606023X.

[29] O.L. Gribkova, O.D. Omelchenko, A.R. Tameev, D.A. Lypenko, A.A. Nekrasov, O.Y. Posudievskii, V.G. Koshechko, A. V. Vannikov, The specific effect of graphene additives in polyaniline-based nanocomposite layers on performance characteristics of electroluminescent and photovoltaic devices, High Energy Chem. 50 (2016) 134-138. doi:10.1134/S001814391602003X.

[30] C. Majumder, A. Rai, C. Bose, Optik Performance optimization of bulk heterojunction organic solar cell, Opt. - Int. J. Light Electron Opt. 157 (2018) 924-929. doi:10.1016/j.ijleo.2017.11.114.

[31] K. Lee, K.H. Cho, J. Ryu, J. Yun, H. Yu, J. Lee, W. Na, J. Jang, Low-cost and efficient perovskite solar cells using a surfactant-modified polyaniline:poly(styrenesulfonate) hole 
transport material, Electrochim. Acta. 224 (2017) 600-607.

doi:10.1016/j.electacta.2016.12.103.

[32] K.-G. Lim, S. Ahn, H. Kim, M.-R. Choi, D.H. Huh, T.-W. Lee, Self-Doped Conducting Polymer as a Hole-Extraction Layer in Organic-Inorganic Hybrid Perovskite Solar Cells, Adv. Mater. Interfaces. 3 (2016) 1500678. doi:10.1002/admi.201500678.

[33] A.H. Fallahpour, A. Gagliardi, D. Gentilini, A. Zampetti, F. Santoni, M. Auf der Maur, A. Di Carlo, Optoelectronic simulation and thickness optimization of energetically disordered organic solar cells, J. Comput. Electron. 13 (2014) 933-942. doi:10.1007/s10825-014-0611-y. 
(a)

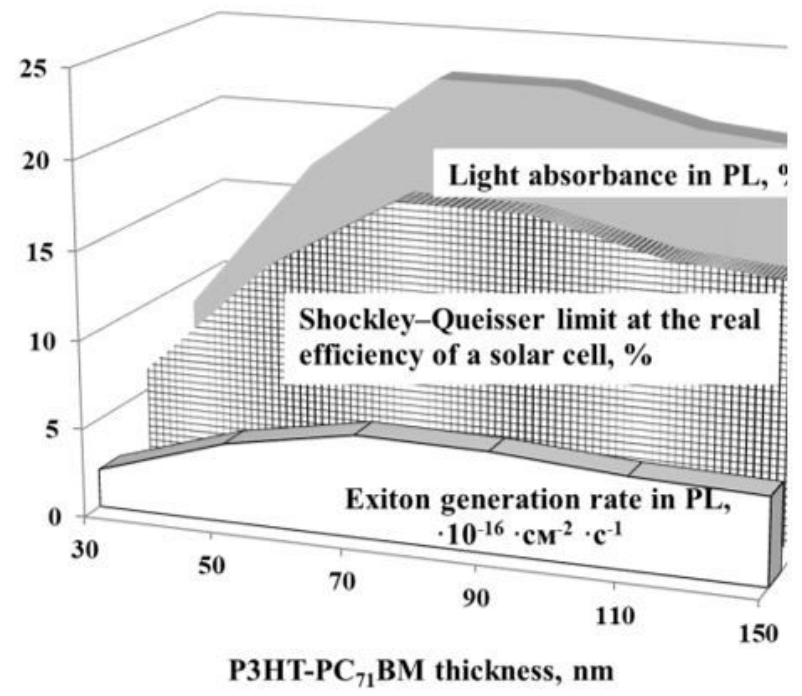

(b)

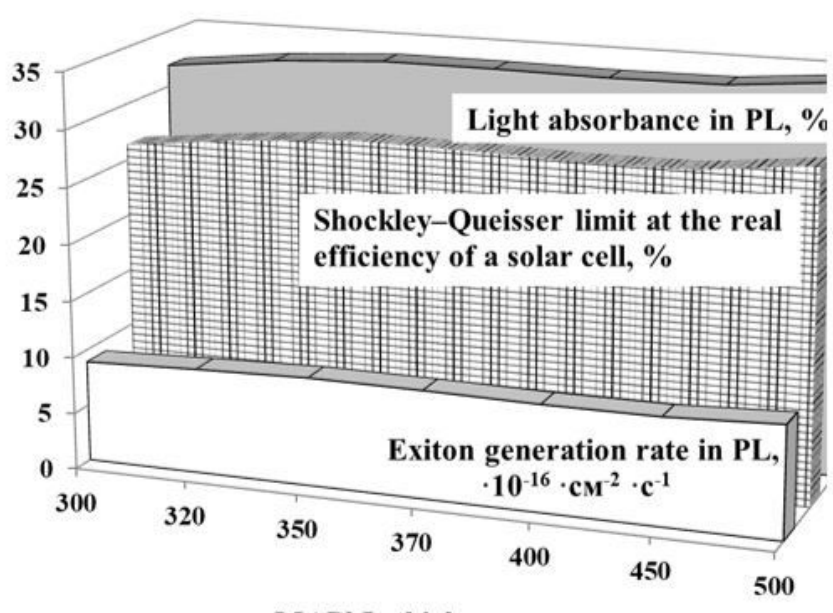

$\mathrm{MAPbI}_{3}$ thickness, nm

Figure 1

Calculated light absorbance in PAL, Shockley-Queisser limit at the real SC efficiency and exciton generation rate in the PAL for varied P3HT-PC71BM (a) and MAPbI3 (b) thickness. 